\section{FRI0069 NEUTROPHILS PLAY A KEY ROLE IN THE REGULATION OF THE CHRONIC INFLAMMATION ASSOCIATED WITH RHEUMATOID ARTHRITIS THROUGH EPIGENETIC MECHANISMS MODULATED BY ANTI-CCPS ANTIBODIES AND REVERSED BY BIOLOGIC THERAPIES}

P. Ruiz-Limon, I. Arias de la Rosa, M.C. Abalos, C. Perez-Sanchez, Y. Jimenez-Gomez, M. Caracuel, J. Calvo, R. Ortega, M. Castro, E. Collantes, A. Escudero, C. Lopez-Pedrera, N. Barbarroja. Rheumatology Service, IMIBIC/Reina Sofia Hospital/University of Cordoba, Cordoba, Spain

Background: MicroRNAs (miRNA) are as a new class of modulators of gene expression, regulating inflammation, degradation of extracellular matrix and invasive behavior of the resident cells in rheumatoid arthritis (RA).

Objectives: 1) To investigate the miRNA expression profile in synovial and blood neutrophils in RA and its role in the pathogenesis of this disorder. 2) To study the effects of biological therapies on the miRNA profile in neutrophils.

Methods: Neutrophils were isolated from peripheral blood (PB) of 25 healthy donors (HD) and 25 RA patients. Neutrophils were isolated from paired synovial fluid (SF) of 15 RA patients. nCounter microRNA Assay was used to detect 800 human microRNAs simultaneously. Altered miRNAs were analyzed for potential mRNA targets using Ingenuity pathways analysis (IPA) software. mRNA targets and genes involved in miRNA biogenesis were evaluated. RA neutrophils were treated in vitro with tocilizumab and infliximab. Healthy neutrophils were treated in vitro with anti-CCPs isolated from RA patients alone or combined with tocilizumab or infliximab. RA neutrophils were cotransfected with the pre-miRNAs 126 and 148.

Results: 94 miRNAs were downregulated and 3 upregulated in PB neutrophils from RA patients compared to HD.Among the miRNAs deregulated in blood, 34 miRNAs were even more significantly reduced in SF neutrophils. Accordingly, RA neutrophils showed a downregulation of the proteins participating in miRNA biogenesis and upregulation of its mRNA targets. Altered miRNAs were mainly involved in immunological disease and inflammatory response, suggesting the abnormal activation of this cell subtype in RA. In vitro treatment of RA neutrophils with infliximab reversed the expression of miRNAs, genes involved in their biogenesis and reduced the inflammatory profile in these cells.In addition, treatment of healthy neutrophils with IgGs anti-CCPs isolated from RA patients decreased the expression of the miRNAs, proteins involved in the biogenesis machinery and increase its inflammatory targets. Finally, cotransfection of RA neutrophils with the pre-miRNAs 126 and 148 reduced the levels of their target proteins.

Conclusions: 1)RA neutrophils exhibit a defect in the miRNAs processing, showed by a decrease in most of the detected miRNAs and the downregulationof proteins involved in their biogenesis. This defect seems to be modulatedby antiCCPs antibodies.2)miRNA downregulation is even more pronounced in synovial fluid neutrophils, which may contribute to the high inflammatory profile of these cells in the joint. 3) Infliximab reverses the altered miRNA profile and the defect in the biogenesis machinery, reducing the proinflammatory pattern in these cells. 4)miRNAs 126 and 148 regulate the expression of numerous inflammatory proteins in RA neutrophils.In sum, this study shows that the neutrophils play a key role in the regulation of the chronic inflammation link to RA. The effect, associated to epigenetic mechanisms, seems to be mediated by the anti-CCPs and is reversed by biologic therapies.

Acknowledgements: Funded by CTS7940, PI2013-0191, ISCIII (CP15/00158, PI15/001333), RD16/0012/0015

Disclosure of Interest: None declared

DOI: 10.1136/annrheumdis-2017-eular.4689

\section{FRI0070 ANGIOTENSIN II TYPE 2 RECEPTOR (AT2R) IS OVEREXPRESSED IN RHEUMATOID ARTHRITIS AND OSTEOARTHRITIS SYNOVIUM AND INCREASES STEADILY WITH INFLAMMATORY STIMULI: A POTENTIAL NEW TARGET FOR PAIN AND ANTI-INFLAMMATORY THERAPIES}

R. Terenzi ${ }^{1}$, M. Manetti $^{2}$, I. Rosa ${ }^{2}$, E. Romano ${ }^{1}$, F. Galluccio ${ }^{1}$, S. Guiducci ${ }^{1}$, L. Ibba-Manneschi ${ }^{2}$, M. Matucci-Cerinic ${ }^{1}$. ${ }^{1}$ Department of Medicine, Division of Rheumatology; ${ }^{2}$ Department of Anatomy, Università degli Studi di Firenze, Firenze, Italy

Background: Despite increasing evidence suggesting that angiotensin II type 2 receptor (AT2R) antagonism might be a feasible strategy for the treatment of chronic inflammation and pain, no study has yet analyzed the synovial expression of AT2R.

Objectives: To investigate the expression of AT2R in rheumatoid arthritis (RA) and osteoarthritis $(\mathrm{OA})$ synovium and its possible modulation in fibroblast-like synoviocytes (FLS) by proinflammatory stimuli.

Methods: The expression of AT2R in RA and OA synovium was investigated by immunohistochemistry. AT2R expression in synovial T cells, B cells, macrophages and FLS was assessed by double immunofluorescence. FLS were isolated from healthy $(\mathrm{H}), \mathrm{OA}$ and RA synovium and treated with tumor necrosis factor (TNF)- $\alpha$ and interleukin (IL)-1 $\beta$, alone or in combination. Immunocytochemistry and Western blotting were performed to study AT2R expression in cultured FLS. Results: RA synovium showed stronger AT2R immunostaining than OA synovium in the lining and sublining layers. In RA synovium, AT2R was strongly expressed in $\mathrm{CD} 3+\mathrm{T}$ cells, CD20+ B cells, CD68+ macrophages and vimentin+ FLS. High levels of AT2R were found in OA-FLS and RA-FLS at baseline, while AT2R expression was negligible in basal H-FLS. AT2R expression was higher in RA-FLS than OA-FLS. Treatment with TNF- $\alpha$ and IL- $1 \beta$ was able to foster the expression of AT2R not only in OA-FLS and RA-FLS, but also in H-FLS.

Conclusions: AT2R is strongly expressed in different cell types of the inflamed synovium and proinflammatory stimuli may foster the expression of AT2R in FLS. AT2R might represent a novel potential therapeutic target in chronic arthritides.

Disclosure of Interest: None declared

DOI: 10.1136/annrheumdis-2017-eular.1338

\section{FRI0071 TOFACITINIB RESTORES THE INHIBITION OF REVERSE CHOLESTEROL TRANSPORT INDUCED BY INFLAMMATION: UNDERSTANDING THE LIPID PARADOX ASSOCIATED WITH RHEUMATOID ARTHRITIS}

S. Pérez Baos, J.I. Barrasa, P. Gratal, A. Larrañaga-Vera, I. Prieto-Potin, G. Herrero-Beaumont, R. Largo. Bone and Joint Research Unit, Rheumatology Dept., IIS-Fundación Jiménez Díaz UAM, Madrid, Spain

Background: Patients with active rheumatoid arthritis (RA) have increased cardiovascular mortality, paradoxically associated with reduced serum lipid levels. In fact, an inverse relationship between C-reactive protein (CRP) and circulating lipid levels has been observed [1,2]. The Jak inhibitor tofacitinib ameliorates systemic and joint inflammation in RA with a concomitant increase in serum lipids [3].

Objectives: Our aim was to analyze the effect of tofacitinib on the lipid and inflammatory profile of hyperlipidemic rabbits with chronic arthritis (CA), and on the regulation of reverse cholesterol transport (RCT) during chronic inflammation. Methods: New Zealand male rabbits were randomly assigned to three groups: control group ( $n=6)$, chronic arthritic rabbits $(C A, n=9)$ and CA rabbits receiving tofacitinib (CA+TOFA, $n=9$ ). All animals were fed ad libitum with a high-fat diet ( $0.5 \%$ cholesterol, $4 \%$ peanut oil). CA was induced in previously immunized rabbits by administering four intra-articular injections of ovalbumin. A group of rabbits received tofacitinib $(10 \mathrm{mg} / \mathrm{kg} /$ day $)$ for two weeks. All animals were euthanized 6 weeks after challenge, when synovial and serum samples were collected. For in vitro studies, vehicle and ABCA1 siRNA-treated THP-1-derived macrophages were exposed to high lipid concentrations, and then stimulated with interferon gamma (IFN $\gamma$ ) in the presence or absence of tofacitinib, in order to assess their cholesterol efflux capacity and the involved mediators.

Results: CA rabbits showed lower levels of serum TC and LDL-C compared to controls $(p=0.001, p=0.012$ ), while TC/HDL-C ratio was higher in CA+TOFA rabbits when compared to $C A$ animals ( $150 \pm 15$ vs $231 \pm 17, p=0.004)$. Synovial inflammation and CRP levels were increased in CA animals, and a significant reduction was shown in CA+TOFA rabbits in both parameters. We also observed an inverse correlation between serum TC and CRP ( $R=-0.454, p=0.029)$. Tofacitinib was able to reduce the lipid content within synovial macrophages up to a $58 \%(p=0.041)$, although it did not modify synovial macrophage density. In foam macrophages in culture, an inflammatory milieu induced by IFN $\gamma$ further stimulated the intracellular lipid accumulation $(\mathrm{p}=0.041)$ along with a decrease in the protein levels of the nuclear factor LXR $\alpha$ (liver X receptor $\alpha$ ) and the cholesterol transporter ABCA1 (ATP-binding cassette transporter 1) $(p=0.002$ and $p=0.0047$, respectively). Tofacitinib prevented the lipid accumulation within macrophages $(p=0.029)$ by increasing $\operatorname{LXR} \alpha(p=0.047)$ and ABCA1 synthesis $(p=0.004)$ in a Jak/STAT-dependent manner, while it was unable to reduce lipid accumulation in ABCA1 silenced macrophages.

Conclusions: Our results suggest that active inflammation could be associated with lipid accumulation within macrophages - in the synovium and probably in other tissues - thus inducing a decrease in serum lipid levels. Tofacitinib may prevent this phenomenon, at least partially, by increasing RCT pathways in macrophages. Taken together, these findings further explain how serum lipid levels are diminished in RA and partially justify the effect of tofacitinib on the lipid profile in RA patients.

References:

[1] Choy E, Sattar N. Ann Rheum Dis 2009;68:460-9.

[2] Johnsson $\mathrm{H}$ et al. Ann Rheum Dis 2013;73:1495-9.

[3] van Vollenhoven RF et al. N Engl J Med 2012;367:508-19.

Disclosure of Interest: None declared

DOI: 10.1136/annrheumdis-2017-eular.2693

\section{FRI0072 ALTERATION OF THE INTESTINAL MICROBIOME IN THE PRECLINICAL PHASE OF EXPERIMENTAL ARTHRITIS AND THE EFFICACY OF MICROBIOTA MODULATION IN ESTABLISHED ARTHRITIS IN MICE}

R. Rogier ${ }^{1}$, H. Evans-Marin ${ }^{2}$, P. van der Kraan ${ }^{1}$, W. van den Berg ${ }^{1}$ M. Koenders ${ }^{1}$, J. Scher ${ }^{2}$, S. Abdollahi-Roodsaz ${ }^{1,2} .{ }^{1}$ Rheumatology, Radboud University Medical Center, Nijmegen, Netherlands; ${ }^{2}$ Medicine, New York University School of Medicine, New York, United States

Background: The composition of intestinal microbiota is perturbed in patients with new-onset rheumatoid arthritis (RA) and those with chronic RA. However, it is not known whether the changes in the intestinal microbiome precede 